\title{
Nonparametric Identification of Nonlinear Dynamics of Systems Based on the Active Experiment
}

\author{
Magdalena Boćkowska and Adam Żuchowski \\ Szczecin University of Technology \\ Poland
}

\section{Introduction}

Identification is a process (measured experiment and numerical procedure) aiming at determining a quantitative model of the examined object's behaviour. Identification of dynamics is a process which tries to define a quantitative model of variation of system state with time. The goal in the experiment is to measure inputs and outputs of the examined object, the system excitations and reactions. In special cases the model can be treated as a "black box" but it always has to be connected with physical laws and can not be inconsistent with them.

The most commonly used models of system dynamics are differential equations - general nonlinear, partial, often nonlinear ordinary, rarely linear ordinary, additionally nonstationary and with deviated arguments. Sometimes one considers discrete-time models presented in a form of difference equations, which are simplified models of a certain kind. Integral equations, functional equations etc. are models of a different kind.

E If a model structure is a priori known or if it can be assumed, the identification consists in 0 determination of model parameters and it is defined as parametric identification. If the full

Dी model structure or its part is not known, nonparametric identification has to be used. In domain of linear models an equivalence of linear ordinary differential equations is transfer function, transient response or frequency response. They can be obtained using experiments of various types: passive - observation of inputs or outputs without interaction upon object or active - excitation of the examined object by special signals (determined: \$impulse, leap, periodic, a periodic, lottery: white noise, coloured noise, noise with Ð determined spectrum...).

đั Many possibilities lead to a variety of identification methods. In the last decades various 중 identification methods have been developed. Rich bibliography connected with this ฮ็ thematic includes Uhl's work (Uhl, 1997) which describes computer methods of is identification of linear and nonlinear system dynamics, in time domain and also frequency, \& with short characteristic and a range of their applications.

There are many methods of parametric and nonparametric identifications of linear dynamics of systems (Eykhoff, 1980), (Iserman, 1982), (Söderström \& Stoica, 1989). There are

ญ fewer useful methods applied for systems with nonlinear dynamics (Billings \& Tsang, 1992), ○̊ (Greblicki \& Pawlak, 1994), (Haber \& Keviczky, 1999), thereby a presented simple solution 
can be very useful in identification of the structure of a model of nonlinear dynamics of 1 st and higher orders.

The method of identification has to be adapted to planned and possible experiments and the type of the assumed model structure. Active identification is more precise than that which is based on a passive experiment. A parametric identification connects optimisation with the regression method which allows to decrease the influence of disturbances and consequently increases accuracy of the model parameters. The precision of identification depends on the degree of disturbances elimination, errors of measured methods and accuracy of measuring devices.

The input and output are often measured as disturbed signals. The parametric identification for non-linear systems based on the method of the averaged differentiation with correction was introduced in the paper (Boćkowska, 2003). The method of averaged differentiation allows to filter distorted signals and to obtain their derivatives. Thanks to the correction procedure one can obtain such values of the corrected averaged signals and their derivatives, which are very close to their real values.

\section{Averaged differentiation method with correction as regularization filter}

The method of averaged differentiation is a regularization filter which allows to determine useful signals and their derivatives based on the disturbed signals available from measurement. Operation of averaged differentiation can be used to evaluate a derivative of any function of signal and time, and averaged signals can be used in estimation of model parameters. Nevertheless its application is not sufficient to determine nonlinearity such as multiplication or other non-linear functions of derivatives with different order. The problem can be solved through connecting the operation with specially designed procedures of correction. Hence one can obtain such values of the corrected averaged signals and their derivatives, which are very close to their real values and so can be used to determine nonlinearity with different structures, good enough to estimate parameters of nonlinear models.

\subsection{Definition of averaged differentiation method}

If the signal $x(t)$ is passing through the window $\mathrm{g}(\mathrm{v})$ with the width $\pm \mathrm{d}$ starting from the moment $t_{0}$, Fig. 1, then the output of the window is the signal (Kordylewski \& Wach, 1988):

$$
x\left(t_{0}\right)_{g}=\int_{-d}^{d} x\left(t_{0}+v\right) \cdot g(v) d v .
$$

If the function $x(t)$ is differentiable, then it $x\left(t_{0}+v\right)$ can be expanded into Taylor series in the neighbourhood of the point $t_{0}$ :

$$
x\left(t_{0}+v\right)=\sum_{i=0}^{\infty} \frac{v^{i}}{i !} \cdot x^{(i)}\left(t_{0}\right) .
$$

Denoting the moments of the measurement window as:

$$
m_{i}=\int_{-d}^{d} v^{i} \cdot g(v) d v
$$


the window response takes the form:

$$
x\left(t_{0}\right)_{g}=\sum_{i=0}^{\infty} \frac{m_{i}}{i !} \cdot x^{(i)}\left(t_{0}\right)
$$

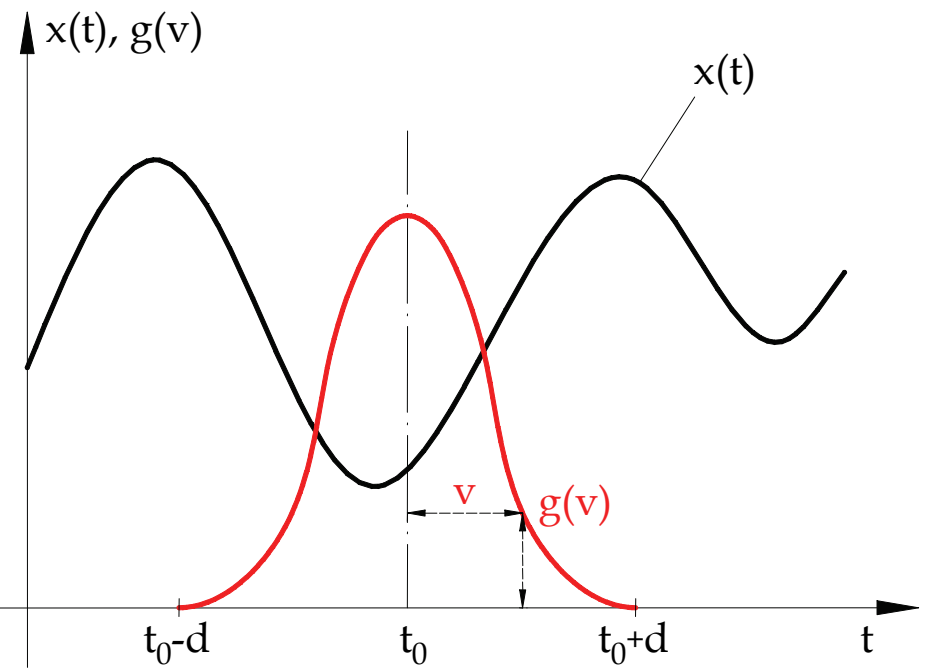

Fig. 1. The measurement window.

In general, we can assume that $x(t)=y^{(n)}(t)$. Using the definition (1) and integrating by parts n-times we get the formula:

$$
\begin{aligned}
& \int_{-d}^{d} y^{(n)}\left(t_{o}+v\right) \cdot g(v)=y^{(n)}\left(t_{o}\right)_{g}=(-1)^{n} \cdot \int_{-d}^{d} y\left(t_{o}+v\right) \cdot g^{(n)}(v) d v+ \\
& \sum_{i=0}^{n-1}(-1)^{i} \cdot\left\{g^{(i)}(d) \cdot y^{(n-i-1)}\left(t_{o}+d\right)-g^{(i)}(-d) \cdot y^{(n-i-1)}\left(t_{o}-d\right)\right\} .
\end{aligned}
$$

If the weight function $\mathrm{g}(\mathrm{v})$ satisfies the following conditions:

$$
g^{(i)}(-d)=g^{(i)}(d)=0 \text { for } i=0,1,2 \ldots n \geq n-1,
$$

then Equation (5) can be simplified to the following form:

$$
y^{(n)}\left(t_{o}\right)_{g}=(-1)^{n} \int_{-d}^{d} y\left(t_{o}+v\right) \cdot g^{(n)}(v) d v .
$$

It allows shifting the differentiation from the signal $y(t)$, which is usually disturbed, to the weight function, which is known in an analytical form. In general, this considerably lowers the influence of disturbances on the result of differentiation, all the stronger the greater the 
measuring window width 2d (Boćkowska, 1998). So the operation of the averaged differentiation can be treated as an equivalent of passing the signal through the measuring window described by the equation:

$$
\mathrm{g}_{\mathrm{n}}(\mathrm{t})=\mathrm{g}^{(\mathrm{n})}(\mathrm{t}) \cdot(-1)^{\mathrm{n}}
$$

The symmetry and normalisation of the function $\mathrm{g}(\mathrm{v})$ are not necessary, but satisfying these conditions is useful for further considerations. Since the function $\mathrm{g}(\mathrm{v})$ is an even function

$$
g(t)=g(-t), \text { for } t \in\langle-d, d\rangle
$$

the odd moments are always equal to zero $m_{2 i+1}=0$ and there are no phase shifts between signals $x\left(t_{0}\right)$ and $x\left(t_{0}\right)_{g}$. If the normalising condition is satisfied:

$$
\int_{-d}^{d} g(v) d v=1
$$

then the transformation (1) corresponds to the averaging of the signal $x(t)$ with the weight function $\mathrm{g}(\mathrm{v})$ in the time interval $\left\langle\mathrm{t}_{0}-\mathrm{d}, \mathrm{t}_{0}+\mathrm{d}\right\rangle$ :

$$
x\left(t_{0}\right)_{g}=\frac{\int_{-d}^{d} x\left(t_{0}+v\right) \cdot g(v) d v}{\int_{-d}^{d} g(v) d v}
$$

\subsection{Optimization of realization of averaged differentiation (Boćkowska, 2005)}

The operation (1) as well as (7) can be interpreted as a convolution conducted in a continuous time domain and it is called the convolution integral. Its value at an arbitrary time $t$ is found by integrating the weighted signal by the window $g(v)$ or its derivative in the averaging interval $(-d, d)$. It can be implemented using one of the numerical integration methods with the fixed step being equal to the measuring step $T_{s}$ or its multiple. Each of them introduces their own errors. The smaller the step size, the more accurate the results. For any given step size, the more computationally complex the method, the more accurate but longer the calculation.

It can be much easier to handle this problem with discrete techniques. The convolution integral can be substituted by the convolution sum:

$$
y_{z}^{(i)}\left(l_{o}\right)_{g}=\sum_{j=0}^{M-1} h(j) \cdot y_{z}\left(l_{o}-j\right), \text { where } h(j)=(-1)^{i} \cdot \frac{g^{(i)}(v)}{T_{s}}
$$

and $\mathrm{M}, \mathrm{l}_{\mathrm{o}}$ are accordingly $2 \cdot \mathrm{d}, \mathrm{t}_{\mathrm{o}}$ divided by the measuring step $\mathrm{T}_{\mathrm{s}}$. If $\mathrm{y}_{\mathrm{z}}$ is the measuring signal with $\mathrm{N}$ samples the result of the convolution, algebraically the same operation as multiplying the polynomials whose coefficients are the elements of signal and window vectors, is N+M-1 point signal, where the first and last $\mathrm{M}$ points may not be useable. The 
time of completing the convolution in the time domain is directly proportional to the width of the window.

FFT convolution uses the overlap-add method together with the FFT procedure and the principle that multiplication in the frequency domain corresponds to convolution in the time domain and reverse. This operation can be written down as:

$$
y_{z}^{(i)}=\operatorname{ifft}\left(f f t(h, M) \cdot f f t\left(y_{z}, M\right)\right)
$$

It is faster than standard convolution for the window with the averaging interval longer than 64 points, because its time increases very slowly, only as the logarithm of the window width M. It produces exactly the same result as the corresponding convolution in the time domain and can be more precise, because the round-off error depends on the total number of calculations, which is directly proportional to the computation time. The FFT convolution called high-speed convolution is the best way to complete the operation of the averaged differentiation.

\subsection{Form of measurement window and degree of noise attenuation}

As a weight function $\mathrm{g}(\mathrm{v})$ one can use any even function of the form:

$$
\mathrm{g}(\mathrm{v})=\mathrm{k}(\mathrm{n}) \cdot \mathrm{f}_{1}(\mathrm{v}) \cdot\left[\mathrm{f}_{2}(\mathrm{v})\right]^{\mathrm{n}}
$$

if a function $\mathrm{f}_{2}(\mathrm{v})$ is even and satisfies the condition $\mathrm{f}_{2}( \pm d)=0$, and $\mathrm{k}(\mathrm{n})$ is the normalising coefficient. Making the Fourier transformation of the window $g(v)$ one gets the window spectrum $G(j \omega)$ :

$$
G(j \omega)=\int_{-d}^{d} g(v) \cdot \exp (-j \omega v) d v .
$$

Since the function $\mathrm{g}(\mathrm{v})$ is even its Fourier spectrum contains no imaginary part and can be written in the form:

$$
G(j \omega)=\operatorname{Re} G(j \omega)=2 \cdot \int_{0}^{d} g(v) \cdot \cos (\omega v) d v .
$$

It means that the operation of averaging does not introduce any phase. If to a weight function $g(v)$ we assign the log magnitude function:

$$
G_{L}(\omega)=20 \cdot \log _{10}\left|\int_{-d}^{d} g(v) \cdot \exp (-j \omega v) d v\right|,
$$

then $n$-th derivative $\mathrm{g}^{(\mathrm{n})}(\mathrm{v})$ corresponds to:

$$
G_{L}(\omega)_{n}=20 \cdot \log _{10}\left|\omega^{n} \cdot \int_{-d}^{d} g(v) \cdot \exp (-j \omega v) d v\right| .
$$


The normalised frequency is introduced for generality:

$$
\Omega=\omega \cdot \frac{2 \cdot \mathrm{d}}{\pi} \text {. }
$$

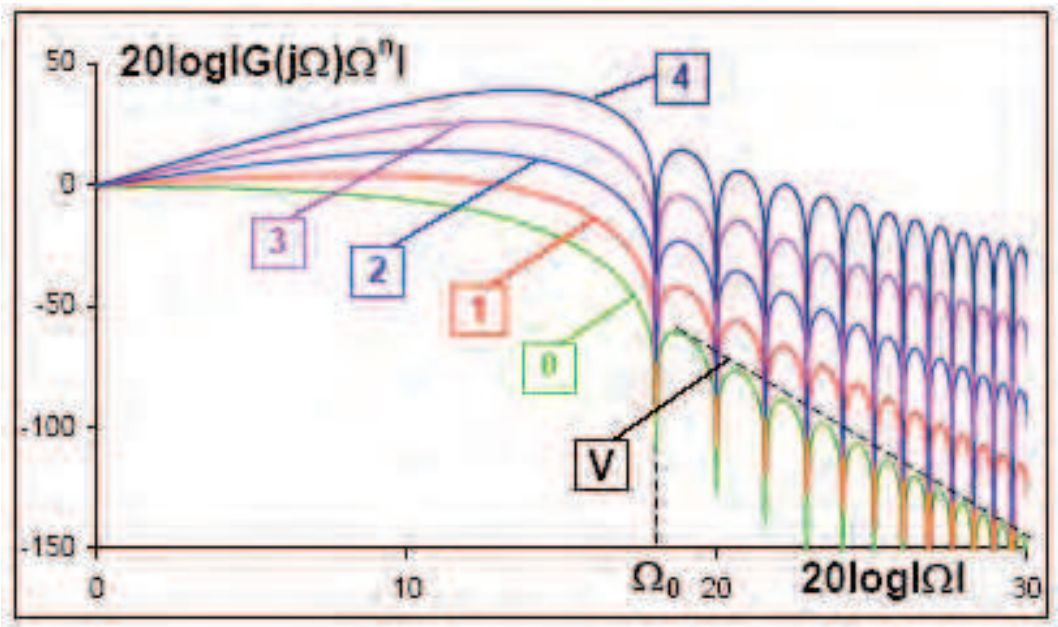

Fig. 2. The logarithmic characteristics of the derivative spectrum of the Nutall window.

The exemplary Bode plots in the logarithmic scale $\mathrm{G}_{\mathrm{L}}(\Omega)_{\mathrm{n}}$ for the weight function:

$$
\mathrm{g}(\mathrm{v})=\frac{\mathrm{k}(\mathrm{r})}{\mathrm{d}} \cdot \cos ^{\mathrm{r}}\left(\frac{\pi \cdot \mathrm{v}}{2 \cdot \mathrm{d}}\right)
$$

which corresponds to the Nuttall window (Nuttall, 1981) with $r=6$ and the normalising coefficients $\mathrm{k}(6)=8 / 5$, are shown in Fig. 2 . The plots consist of several lobes and because of the normalisation (10) we have $G(0)=1$ and $G_{L}(0)_{n}=0$. Let $\Omega_{0}$ denote the width of the first lobe and $\mathrm{V}$ denote the averaged rate of the decrease of $\mathrm{G}_{\mathrm{L}}$ except for the first lobe. The correct reconstruction of the signal $\times\left(\mathrm{t}_{0}\right)_{\mathrm{g}}$ takes place in the frequency band of the initial part of the main lobe to $\Omega_{0}$ while the disturbance attenuation - in the frequency band of secondary lobes.

If the signal $y\left(t_{0}\right)$ has the spectrum $Y(j \Omega)$, then the spectrum of the averaged signal $y\left(t_{0}\right)_{g}$ has the following form:

$$
\mathrm{Y}(\mathrm{j} \Omega)_{\mathrm{g}}=\mathrm{Y}(\mathrm{j} \Omega) \cdot \mathrm{G}(\mathrm{j} \Omega) .
$$

The error caused by the process of averaging can be evaluated as the deviation of $G(j \Omega)$ from 1. If $Y(j \Omega)$ and $Z(j \Omega)$ denote the spectra of the signal $y(t)$ and disturbances $z(t)$, respectively, then the operation of averaging differentiation of the disturbed signal $\mathrm{y}_{\mathrm{z}}(\mathrm{t})=\mathrm{y}(\mathrm{t})+\mathrm{z}(\mathrm{t})$ is expressed by the equation:

$$
y_{z}^{(n)}\left(t_{0}\right)_{g}=(-1)^{n} \cdot \int_{-d}^{d} y\left(t_{0}+v\right) \cdot g^{(n)}(v) d v+(-1)^{n} \cdot \int_{-d}^{d} z\left(t_{0}+v\right) \cdot g^{(n)}(v) d v,
$$


which illustrates the process of reducing the effect of disturbances at an adequately selected window $\mathrm{g}(\mathrm{v})$. It corresponds to the spectrum:

$$
Y_{z}(j \Omega)=(j \times \Omega)^{n} \times Y(j \Omega) \times G(j \Omega)+(j \times \Omega)^{n} \times Z(j \Omega) \times G(j \Omega) .
$$

If the function $y^{(n)}(t)$ is continuous and differentiable, then we can use its expansion in Taylor series taking into consideration the conditions (6) and (9). Hence we obtain:

$$
y^{(n)}\left(t_{0}\right)_{g}=y^{(n)}\left(t_{0}\right)+\sum_{i=1}^{\infty} \frac{m_{2 i}}{(2 \cdot i) !} \cdot y^{(n+2 i)}\left(t_{0}\right)+(-1)^{n} \cdot \int_{-d}^{d} z\left(t_{0}+v\right) \cdot g^{(n)}(v) d v
$$

This proves that the deformation of the signal $y^{(n)}\left(t_{0}\right)_{g}$ with regard to the derivative $y^{(n)}\left(t_{0}\right)$ has two components: the first is caused by averaging and the second by the presence of disturbances:

$$
\mathrm{y}^{(\mathrm{n})}\left(\mathrm{t}_{0}\right)_{\mathrm{g}}-\mathrm{y}^{(\mathrm{n})}\left(\mathrm{t}_{0}\right)=\Delta_{1}+\Delta_{2} .
$$

The first error $\Delta_{1}$ depends on the smoothness of the signal $\mathrm{y}^{(\mathrm{n})}\left(\mathrm{t}_{0}\right)$ and on the values of the even moments of the weight function, which for the Nuttall window are given by:

$$
m_{2 j}=d^{2 \cdot j} \cdot \Theta(2 j, r),
$$

where $\Theta$ are the constant coefficients for the function $g(v)$ in the given form. So it follows that the error $\Delta_{1}$ can be diminished by decreasing of $\mathrm{d}$ or by a suitable choice of the form of weight function $g(v)$, which allows to decrease coefficients $\Theta$. Because of truth of the inequality:

$$
\int_{-d}^{d}\left\{z\left(t_{0}+v\right)\right\}^{2} \cdot\left\{g^{(n)}(v)\right\}^{2} d v \leq\left\{z\left(t_{0}+v\right)\right\}_{\max }^{2} \cdot \int_{-d}^{d}\left\{g^{(n)}(v)\right\}^{2} d v
$$

the influence of disturbances can be evaluated by the following measure:

$$
M_{n}=\sqrt{\int_{-d}^{d}\left\{g^{(n)}(v)\right\}^{2} d v}=\frac{\Psi(n, r)}{d^{n+0.5}}
$$

where $\Psi$ is the characteristic coefficient of the weight function $g(v)$, which is growing with the increase of the derivative order $n$ very quickly. The reduction of this error can be done by increasing of $\mathrm{d}$ and by a suitable choice of the form of the weight function $\mathrm{g}(\mathrm{v})$. So it follows that the greater $\mathrm{d}$ the greater $\Delta_{1}$ and simultaneously the less $\Delta_{2}$. The interval of averaging and the form of the weight function $\mathrm{g}(\mathrm{v})$ should be selected so as to minimise both of these errors. The minimisation of considered errors can be done by a suitable choice of the weight function $g(v)$ so that coefficients $\Theta$ and $\Psi$ should be possibly small.

\subsection{Designing optimal correction procedure (Boćkowska, 2006)}

The form of the known weight function can be corrected through the application of special procedures, which can correspond to the following operation: 


$$
y\left(t_{o}\right)_{k}=C_{0} \cdot y\left(t_{o}\right)_{g}+\frac{1}{2} \sum_{i=1}^{n} C_{i} \cdot\left[y\left(t_{o}+T_{i}\right)_{g}+y\left(t_{o}-T_{i}\right)_{g}\right]
$$

Expanding signals $y_{g}$ in the Taylor series we obtain the relation:

$$
\begin{aligned}
& y\left(t_{o}\right)_{k}=y\left(t_{o}\right) \cdot\left(C_{0}+C_{1}+C_{2}+\ldots\right)+ \\
& y^{(2)}\left(t_{o}\right) \cdot\left\{m_{2} \cdot\left(C_{0}+C_{1}+C_{2}+\ldots\right) / 2 !+C_{1} \cdot T_{1}^{2}+C_{2} \cdot T_{2}^{2}+\ldots\right\}+ \\
& y^{(4)}\left(t_{o}\right) \cdot\left\{m_{4} \cdot\left(C_{0}+C_{1}+\ldots\right)+4 ! \cdot m_{2} \cdot\left(C_{1} \cdot T_{1}^{2}+\ldots\right) / 2 ! \cdot 2 !+C_{1} T_{1}^{4}+C_{2} T_{2}^{4}+\ldots\right\} / 4 !+ \\
& y^{(6)}\left(t_{o}\right) \cdot\left\{m_{6} \cdot\left(C_{0}+C_{1}+C_{2}+\ldots\right)+6 ! \cdot m_{4} \cdot\left(C_{1} \cdot T_{1}^{2}+C_{2} \cdot T_{2}^{2}+\ldots\right) / 2 ! \cdot 4 !+\right. \\
& \left.6 ! \cdot m_{2} \cdot\left(C_{1} \cdot T_{1}^{4}+C_{2} \cdot T_{2}^{4}+\ldots\right) / 4 ! \cdot 2 !+C_{1} \cdot T_{1}^{6}+C_{2} \cdot T_{2}^{6}+\ldots\right\} / 6 !+\ldots
\end{aligned}
$$

Because of $y_{k}\left(t_{o}\right) \cong y\left(t_{o}\right)$ the evaluation of the corrector parameters $C_{i}$ and $T_{i}$ should be done on the basis of the following system of non-linear equations:

$$
\begin{aligned}
& C_{0}+C_{1}+\ldots+C_{n}=1 \\
& C_{1} \cdot T_{1}^{2}+C_{2} \cdot T_{2}^{2}+\ldots+C_{n} \cdot T_{n}^{2}=2 ! \cdot M_{2}=A_{2} \\
& C_{1} \cdot T_{1}^{4}+C_{2} \cdot T_{2}^{4}+\ldots+C_{n} \cdot T_{n}^{4}=4 ! \cdot\left(M_{2}^{2}-M_{4}\right)=A_{4} \\
& C_{1} \cdot T_{1}^{6}+C_{2} \cdot T_{2}^{6}+\ldots+C_{n} \cdot T_{n}^{6}=6 ! \cdot\left(M_{2}^{3}-2 \cdot M_{2} \cdot M_{4}+M_{6}\right)=A_{6} \\
& C_{1} \cdot T_{1}^{8}+C_{2} \cdot T_{2}^{8}+\ldots+C_{n} \cdot T_{n}^{8}=8 ! \cdot\left(M_{2}^{4}+2 \cdot M_{2} \cdot M_{6}+M_{4}^{2}-3 \cdot M_{2}^{2} \cdot M_{4}-M_{8}\right)=A_{8} \\
& C_{1} \cdot T_{1}^{10}+C_{2} \cdot T_{2}^{10}+\ldots+C_{n} \cdot T_{n}^{10}=10 ! \cdot\left(M_{2}^{5}-2 \cdot M_{2} \cdot M_{8}-2 \cdot M_{4} \cdot M_{6}+3 \cdot M_{2}^{2} \cdot M_{6}+\right. \\
& \left.3 \cdot M_{2} \cdot M_{4}^{2}-4 \cdot M_{4} \cdot M_{2}^{3}+M_{10}\right)=A_{10}
\end{aligned}
$$

The system of Equations (31) is non-linear with unknowns $C_{i}, T_{i}$ and it is not clear that it has real solutions for arbitrary $\mathrm{n}$ and a given window.

In the identification of the system dynamics we want to obtain such values of the corrected averaged signal and their derivatives, which are possibly close to their real values. The corrected signals should be useful to obtain for example the following nonlinearities:

$$
y^{(n)}(t) \cdot y^{(k)}(t) \cong y_{k}^{(n)}(t) \cdot y_{k}^{(k)}(t)
$$

where $y_{k}^{(n)}(t)$ and $y_{k}^{(n)}(t)$ are set on the basis of the measured signal $y(t)$. To meet this goal possibly more equations from system (31) must be fulfilled. The first consecutive equations allow to obtain the unknowns $C_{i}$ (Boćkowska, 2003). The evaluation of the time constants $T_{i}$ must be done minimizing the rest of equations for a given form of the window, measuring step and the used width $d$ of the averaging interval. The time constants $T_{i}$ can be expressed depending on the width of the averaging interval $\mathrm{d}$ as:

$$
\mathrm{T}_{\mathrm{i}}=\mathrm{T}_{\mathrm{di}} \cdot \mathrm{d}
$$

so $\mathrm{T}_{\mathrm{di}}$ and corresponding $\mathrm{C}_{\mathrm{i}}$ are the corrector parameters. The correction procedure must be unique for different kinds of windows and their chosen order and width. The course of the correction will be considered for the cosine window known as the Max Rolloff 4-Term or Nuttall window of $6^{\text {th }}$ order and the correction procedure of $2^{\text {nd }}$ order in the form: 


$$
\mathrm{y}_{\mathrm{k}}\left(\mathrm{t}_{0}\right)=\mathrm{C}_{0} \cdot \mathrm{y}\left(\mathrm{t}_{0}\right)_{\mathrm{g}}+\frac{1}{2} \sum_{\mathrm{i}=1}^{2} \mathrm{C}_{\mathrm{i}} \cdot\left[\mathrm{y}\left(\mathrm{t}_{0}+\mathrm{T}_{\mathrm{i}}\right)_{\mathrm{g}}+\mathrm{y}\left(\mathrm{t}_{0}-\mathrm{T}_{\mathrm{i}}\right)_{\mathrm{g}}\right] .
$$

The corrector parameters $C_{i}$ are determined by the following relations:

$$
\begin{aligned}
& \mathrm{C}_{1}=\left(\mathrm{A}_{2} \cdot \mathrm{T}_{2}^{2}-\mathrm{A}_{4}\right) /\left(\mathrm{T}_{1}^{2} \cdot \mathrm{T}_{2}^{2}-\mathrm{T}_{1}^{4}\right) \\
& \mathrm{C}_{2}=\left(\mathrm{A}_{4}-\mathrm{A}_{2} \cdot \mathrm{T}_{1}^{2}\right) /\left(\mathrm{T}_{2}^{4}-\mathrm{T}_{1}^{2} \cdot \mathrm{T}_{2}^{2}\right) \\
& \mathrm{C}_{0}=1-\mathrm{C}_{1}-\mathrm{C}_{2}
\end{aligned}
$$

which follow from the first three equations of the system (31). The determination of the time constants $T_{i}$ requires optimisation. After introduction of two auxiliary variables:

$$
\mathrm{x}=\mathrm{T}_{1}^{2} \text { and } \mathrm{y}=\mathrm{T}_{2}^{2}
$$

the next equation can be written as a relation, which is linear and symmetrical in regards to $x$ and $y$ :

$$
f(x, y)=y \cdot A_{4}-y \cdot x \cdot A_{2}+x \cdot A_{4}-A_{6}=0
$$

As it is shown in Figure 3 Equation (37) is never satisfied for the positive $\mathrm{x}$ and $\mathrm{y}$ and has to be omitted. The fifth equation from the system (31) is a quadratic equation with two roots:

$$
g(x, y)=y^{2} \cdot A_{4}-y^{2} \cdot x \cdot A_{2}+y \cdot x \cdot A_{4}-y \cdot x^{2} \cdot A_{2}+x^{2} \cdot A_{4}-A_{8}=0
$$

The positive one can be taken into consideration. It can be written as the next function:

$$
\begin{aligned}
& x(y)=\left[-y^{2} \cdot A_{2}+y \cdot A_{4}-\left(y^{4} \cdot A_{2}^{2}+2 \cdot y^{3} \cdot A_{2} \cdot A_{4}-3 \cdot y^{2} \cdot A_{4}^{2}\right.\right. \\
& \left.\left.-4 \cdot y \cdot A_{2} \cdot A_{8}+4 \cdot A_{4} \cdot A_{8}\right)^{1 / 2}\right] / 2 \cdot\left(y \cdot A_{2}-A_{4}\right)
\end{aligned}
$$

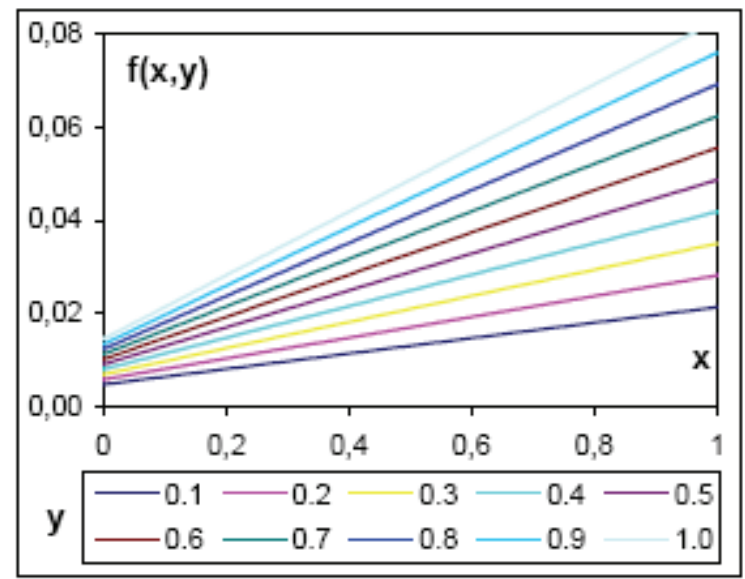

Fig. 3. Relation $f(x, y)$ defined by Equation (37). 


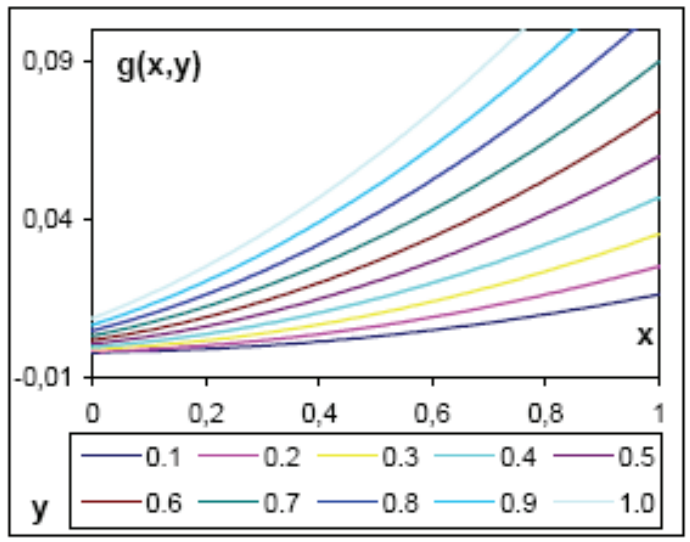

Fig. 4. Relation $g(x, y)$ defined by Equation (38).

which allows us to obtain the allowed interval of the variability of $y$ and $x$, because their changes are symmetrical. The additional condition is needed to obtain both $\mathrm{x}$ and $\mathrm{y}$ :

$$
\left|\mathrm{C}_{0}\right|+\left|\mathrm{C}_{1}\right|+\left|\mathrm{C}_{2}\right|=\min
$$

which follows from the postulate of the minimal increase of the disturbances participation. Taking into consideration (35) and (39) it can be written down as the function of y:

$$
h(y)=\left|\frac{y \cdot A_{2}-A_{4}}{x(y) \cdot(y-x(y))}\right|+\left|\frac{x(y) \cdot A_{2}-A_{4}}{y \cdot(y-x(y))}\right|+\left|\frac{x(y) \cdot A_{2}-A_{4}+y \cdot A_{2}-x(y) \cdot y}{x(y) \cdot y}\right|
$$

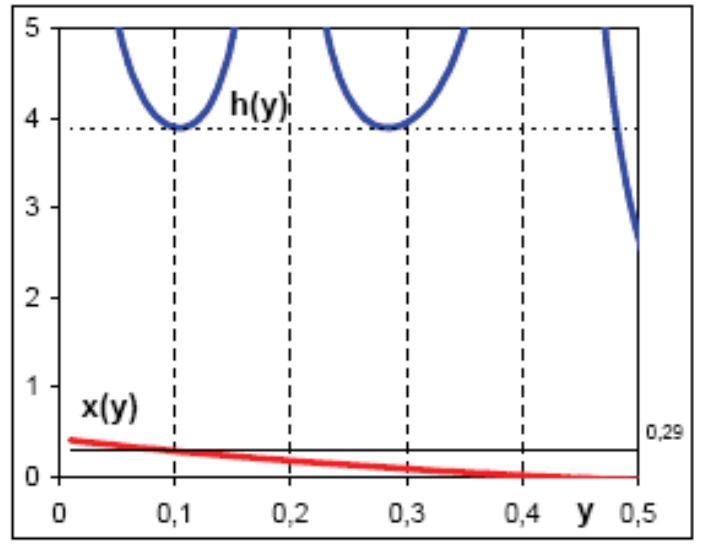

Fig. 5. Variability of the auxiliary variable $\mathrm{x}$ and function $\mathrm{h}(\mathrm{y})$.

The above derivation is valid for corrector of $2^{\text {nd }}$ order of any window, but presented graphs correspond to Nuttall window of $6^{\text {th }}$ order. The determined values of the coefficients $A_{2,4,6,8}$ and corrector parameters $C_{0,1,2}$ and $T_{1,2}$ for the Nuttall window of $1^{\text {st }}$ to $6^{\text {th }}$ order are shown in Table 1. The presented values are obviously functions of the window width $\mathrm{d}$. 


\begin{tabular}{|c|c|c|c|c|c|c|c|c|c|}
\hline$R$ & $A_{2}$ & $A_{4}$ & $A_{6}$ & $A_{8}$ & $C_{0}$ & $C_{1}$ & $C_{2}$ & $T_{d 1}$ & $T_{d 2}$ \\
\hline $\mathbf{1}$ & -0.189 & 0.137 & -0.207 & 0.547 & 1.773 & 0.21 & -0.983 & 1.139 & 0.686 \\
\hline $\mathbf{2}$ & -0.131 & 0.061 & -0.058 & -0.091 & 1.878 & 0.251 & -1.129 & 0.89 & 0.54 \\
\hline $\mathbf{3}$ & -0.099 & 0.034 & -0.023 & 0.025 & 1.977 & 0.265 & -1.242 & 0.75 & 0.447 \\
\hline $\mathbf{4}$ & -0.08 & 0.022 & -0.011 & 0.009 & 2.013 & 0.316 & -1.329 & 0.648 & 0.4 \\
\hline $\mathbf{5}$ & -0.067 & 0.015 & -0.006 & 0.004 & 2.108 & 0.29 & -1.398 & 0.589 & 0.346 \\
\hline $\mathbf{6}$ & -0.057 & 0.011 & -0.004 & 0.002 & 2.119 & 0.330 & -1.449 & 0.533 & 0.323 \\
\hline
\end{tabular}

Table 1. The coefficients A and corrector parameters $C$ and $T$ for the Nuttall window of $1^{\text {st }}$ to $6^{\text {th }}$ order.

Figure 5 shows the variability of the slack variables $\mathrm{x}, \mathrm{y}$ and thereby the time constants $\mathrm{T}_{1}$ and $\mathrm{T}_{2}$. The minimum of function $\mathrm{h}(\mathrm{y})$ is for $\mathrm{y} \cong 0.29$ or 0.10 and consequently $\mathrm{T}_{2} \cong 0.53$ and 0.32. The time constant $T_{1}$ obtained from Equations (41) and (36) is $T_{1}=0.32$ or 0.53 . Because the product of the time constants and the width $\mathrm{d}$ of the averaging interval expressed in the samples must be integer, so they have to be recalculated using the following relation:

$$
\mathrm{T}_{\mathrm{rdi}}=\frac{\operatorname{round}\left(\mathrm{T}_{\mathrm{di}} \cdot \mathrm{d}\right)}{\mathrm{d}}
$$

The correction procedure of $2^{\text {nd }}$ order corresponds to the change of the basic window spectrum to the following form:

$$
G_{z}(\omega)=G(\omega) \cdot G_{k}(\omega)=G(\omega) \cdot\left[C_{0}+\sum_{i=1}^{2} C_{i} \cdot \cos \left(\omega \cdot T_{i}\right)\right]
$$

Introducing the auxiliary variable:

$$
\mathrm{u}=\frac{\pi \cdot \mathrm{v}}{2 \cdot \mathrm{d}}
$$

the definition (15) can be rewritten in the following form:

$$
\mathrm{G}(\Omega)=\frac{\pi}{\mathrm{d}} \cdot \int_{0}^{\pi / 2} \mathrm{~g}(\mathrm{u}) \cdot \cos (\Omega \cdot \mathrm{u}) \mathrm{du}
$$

and the spectrum of the corrected window assumes the form:

$$
\mathrm{G}_{\mathrm{z}}(\Omega)=\frac{\pi}{\mathrm{d}} \cdot\left[\mathrm{C}_{0}+\sum_{\mathrm{i}=1}^{2} \mathrm{C}_{\mathrm{i}} \cdot \cos \left(\Omega \cdot \mathrm{T}_{\mathrm{ui}}\right)\right] \cdot \int_{0}^{\pi / 2} \mathrm{~g}(\mathrm{u}) \cdot \cos (\Omega \cdot \mathrm{u}) \mathrm{du}
$$

The relation (46) allows to obtain the maximum normalised frequency $\Omega_{\alpha}$, which can be transferred with the assumed accuracy $\alpha$ through the corrected window, solving the following equation:

$$
\alpha=1-\mathrm{G}_{\mathrm{z}}(\Omega)
$$

The evaluation of the corrector parameters $C_{i}$ and $T_{i}$ should be done on the basis of two criteria: the minimisation of the deviation $G(j \Omega)$ from 1 in the pass band and the magnitude 
$\Omega^{\mathrm{n}} \mathrm{G}(\mathrm{j} \Omega)$ in the stop band. The application of the procedures is a trial of the window approach to the ideal window with wall spectral properties.

The correct evaluation of the pass band frequency $f_{p}$ is the next important problem, which decides about the correctness of the processing of the measured signals and has big influence on the quality of the identification. The pass band frequency is a filter feature and for a low-pass filter assigns the maximum frequency, which as a component of a useful signal is transferred without deformation. It determines the transfer band frequency being its upper limit. It is a basis to determine the filter parameters and proper choice of corrector parameters, if a valuable information of signal is lost or if a processed signal is deformed by noise. It decides about the credibility of processed signals as the material for identification of dynamics of systems. The pass band frequency $f_{p}$ can be determined on the basis of the output spectrum which is more complex than the input spectrum, because of the influence of the system's dynamics.

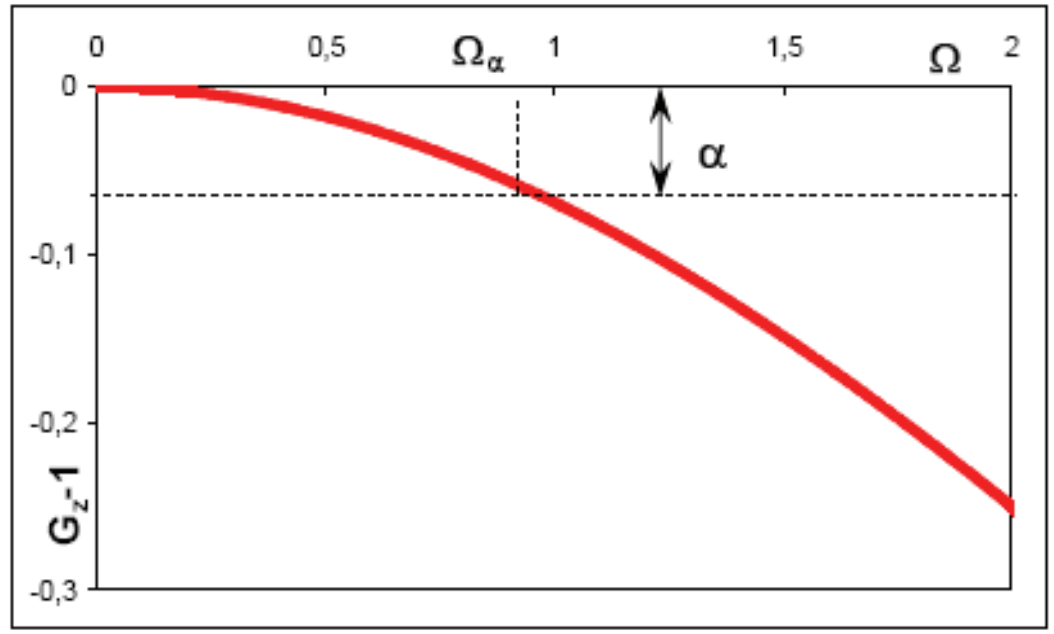

Fig. 6. Accuracy $\alpha$ of transmission of frequencies lower than $\Omega_{\alpha}$ by the corrected window with spectrum $\mathrm{G}_{\mathrm{z}}(\Omega)$.

If a measurement experiment is carried out on the same examined system using the same instruments, but input with different parameters, then one can use the power spectral density of outputs assigned for two extremes of inputs. The power spectral density is suggested in order to increase the spectrum resolution and is defined as follows:

$$
\mathrm{GWM}=\mathrm{Y} \cdot \operatorname{conj}(\mathrm{Y}),
$$

and the frequency axis is defined in $\mathrm{Hz}$ according to $\mathrm{f}=(0: \mathrm{N}-1) /\left(\mathrm{N} \cdot \mathrm{T}_{\mathrm{s}}\right)$, where $\mathrm{N}$ is the number of samples, $\mathrm{T}_{\mathrm{s}}$ - time step between consecutive samples. The frequencies of dominant peaks can be determined through an analysis of the graph of the difference of power spectral density both of outputs.

$$
\Delta \mathrm{G}=\mathrm{GWM}_{1}-\mathrm{GWM}_{2} .
$$

If $\Omega_{\alpha}$ and $f_{p}$ are known then the required width of the measurement window can be calculated according to the following relation: 


$$
\mathrm{d}_{\mathrm{opt}}=\frac{\Omega_{\alpha}}{4 \cdot \mathrm{f}_{\mathrm{p}}}
$$

The determination of $\Omega_{\alpha}$ can be done very easily and quickly if the analytical relation $\Omega_{\alpha}=\mathrm{f}(\alpha)$ fulfilled for small $\alpha$, for example $0,01 \leq \alpha \leq 0,1$, for a given kind of the measurement window is known. An example of such relation for a given window is shown below. Allowing for the correction procedure of $2^{\text {nd }}$ order defined by (34) the spectrum of the corrected window of $r$ order gets the form:

$$
\mathrm{G}(\Omega)=\frac{\left[\frac{2}{\pi \cdot \Omega} \cdot \sin \left(\frac{\pi \cdot \Omega}{2}\right)\right]^{1-\bmod (\mathrm{r}, 2)} \cdot\left[\cos \left(\frac{\pi \cdot \Omega}{2}\right)\right]^{\bmod (\mathrm{r}, 2)} \cdot\left[\mathrm{C}_{0}+\sum_{\mathrm{i}=1}^{2} \mathrm{C}_{\mathrm{i}} \cdot \cos \left(\Omega \cdot \mathrm{T}_{\mathrm{ui}}\right)\right]}{\prod_{\mathrm{n}=1}^{\mathrm{r}}\left[1-\left(\frac{\Omega}{\mathrm{n}}\right)^{2}\right]^{[1-\bmod (\mathrm{r}, 2)-\bmod (\mathrm{n}, 2)]}}
$$

and corresponding plots for $r=1 \div 6$ are shown in Figure 7 .

Figure 8 presents variability of $\Omega_{\alpha}$ versus $\alpha$ in the range $0.01 \leq \alpha \leq 0.1$. The relationship $\Omega_{\alpha}=\mathrm{f}(\alpha)$ in an analytical form is needed to calculate quickly the optimal width of the measurement window $d$ for a given output and its pass band frequency $f_{p}$ according to (51). The approximation of this relation can be evaluated using (51) and (47). The courses in the given range were approximated by an exponential function:

$$
\Omega_{\alpha \text { apr }}=S \cdot \alpha^{\mathrm{P}}
$$

with very good fitting using the regression method. The results: the values of the coefficients $S$ and $P$ as well as correlation coefficients $R$ are shown in Table 2 . The mean value of the exponent $P_{m}$ is equal to 0.197 and was accepted as constant. The new values of coefficients $\mathrm{S}_{\mathrm{m}}$ for $P_{m}$ are also presented in Table 2. For $S_{m}$ and $r$ the quadratic dependence was found as it is shown in Figure 9.

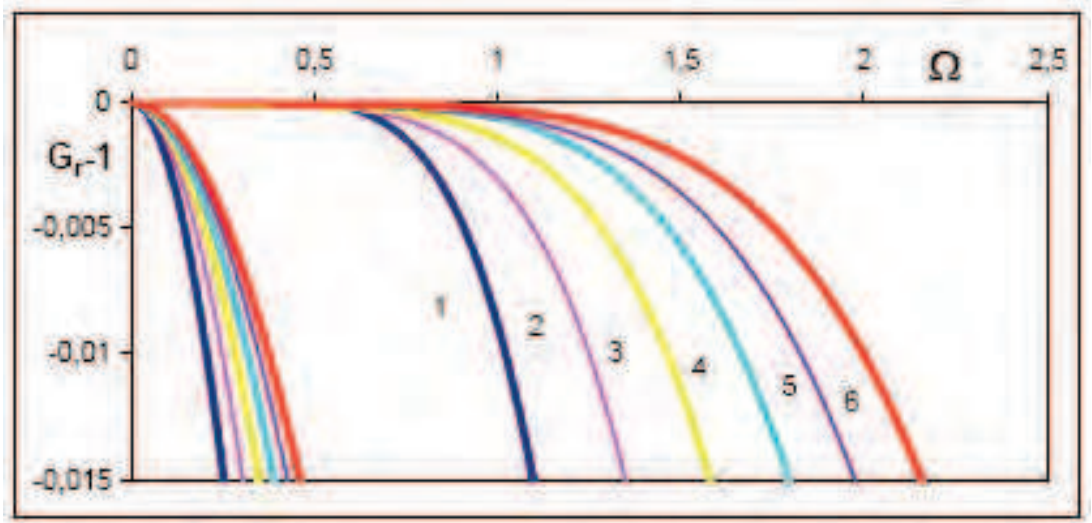

Fig. 7. The spectrum of the corrected Nuttall window of $1^{\text {st }}$ to $6^{\text {th }}$ order versus $\Omega$. 


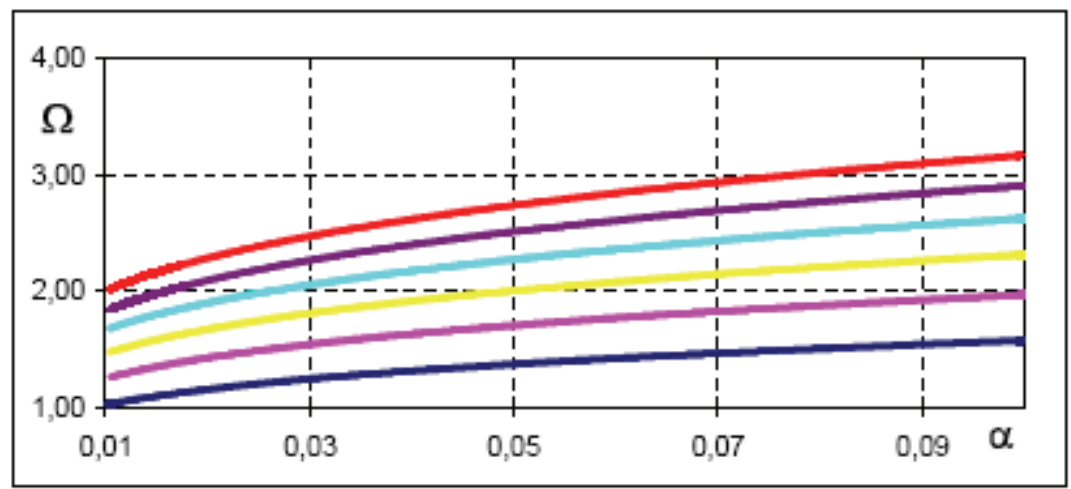

Fig. 8. The normalised frequency $\Omega$ versus the accuracy $\alpha$ of transmission of the useful signal.

\begin{tabular}{|c|c|c|c|c|c|c|}
\hline $\mathbf{r}$ & $\mathbf{1}$ & $\mathbf{2}$ & $\mathbf{3}$ & $\mathbf{4}$ & $\mathbf{5}$ & $\mathbf{6}$ \\
\hline $\mathbf{S}$ & 4.9755 & 4.5838 & 4.1065 & 3.6324 & 3.0745 & 2.4184 \\
\hline $\mathbf{P}$ & 0.1991 & 0.2006 & 0.1971 & 0.1982 & 0.1961 & 0.1882 \\
\hline $\mathbf{R}$ & 0.9997 & 0.9997 & 0.9996 & 0.9997 & 0.9998 & 0.9995 \\
\hline $\mathbf{S}_{\mathbf{m}}$ & 4.941 & 4.529 & 4.105 & 3.618 & 3.083 & 2.49 \\
\hline
\end{tabular}

Table 2. Parameters of the approximate exponential function to the order $r$ of the measurement window.

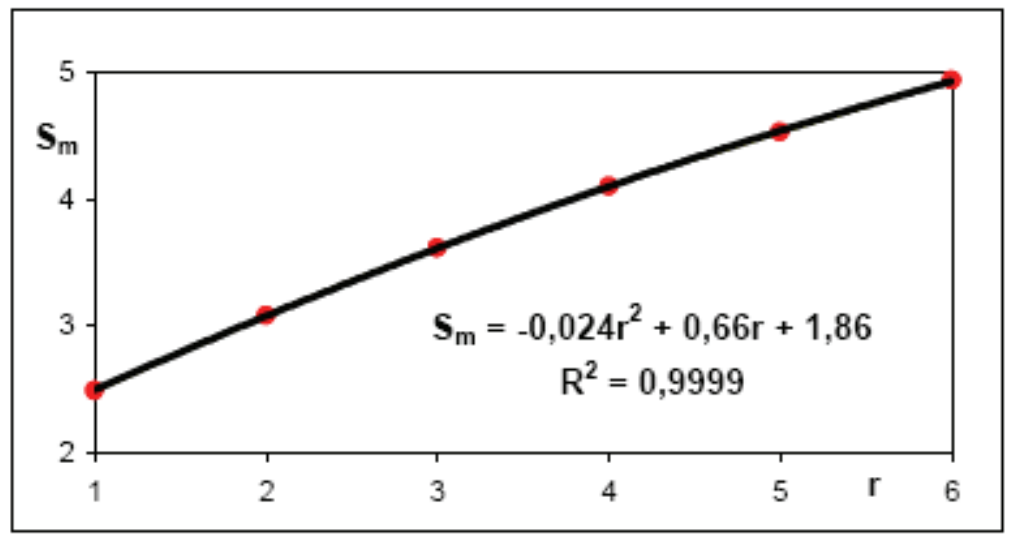

Fig. 9. Variability of $S_{m}$ versus $r$.

The result approximate relation, who determines the variability of $\Omega_{\alpha}$ versus $r$, is as follows:

$$
\Omega_{\alpha \mathrm{apr}}=\left(-0.024 \cdot \mathrm{r}^{2}+0.66 \cdot \mathrm{r}+1.86\right) \cdot \alpha^{0.197}
$$

The correlation coefficients between the data, which was obtained on the basis of the corrected window spectrum and the other calculated on the basis of the above mentioned 
Equation (53), are still close to unity. The needed width of the measurement window can be expressed in seconds, using (54) and measurement step relating to:

$$
\mathrm{d}_{\text {opt }}=\frac{\left(-0.024 \cdot \mathrm{r}^{2}+0.66 \cdot \mathrm{r}+1.86\right) \cdot \alpha^{0.197}}{\left(4 \cdot \mathrm{f}_{\mathrm{p}} \cdot \mathrm{step}\right)} .
$$

\section{Identification of non-linear system dynamics}

\subsection{Parametric identification}

The proposed procedure, the averaged differentiation operation with correction, can be applied to evaluation of the parameters of the model of the system dynamics if the model structure is known and corresponds in general to the differential equations of the kind:

$$
y^{(n)}(t)=F\left(y, y^{(1)}, \ldots, y^{(n-1)}, x, x^{(1)}, \ldots, x^{(n)}, A_{0}, \ldots, B_{0}, \ldots, D\right)
$$

which can be written down as the following equation:

$$
y^{(n)}(t)=\sum_{j=0}^{n} A_{j} \cdot F_{j x}\left(y, \ldots y^{(n-1)}, x, \ldots x^{(n)}\right) \cdot x^{(j)}-\sum_{j=0}^{n-1} B_{j} \cdot F_{j y}\left(y, \ldots y^{(n-1)}, x, \ldots x^{(n)}\right) \cdot y^{(j)}+D
$$

Taking into account all the measured data samples $\mathrm{N}$, to which the model with the parameters $A_{j}, B_{j}$ and $D$ must be fitted we can present the system in the matrix form:

$$
\begin{array}{r}
\mathrm{Yn}=\mathrm{XY} \cdot \mathrm{PAR}, \quad \text { where } \mathrm{Yn}=\left[\begin{array}{c}
\mathrm{y}_{1}^{(\mathrm{n})} \\
\mathrm{y}_{2}^{(\mathrm{n})} \\
\ldots \\
\mathrm{y}_{\mathrm{N}}^{(\mathrm{n})}
\end{array}\right], \mathrm{PAR}=\left[\begin{array}{c}
\mathrm{A}_{0} \\
\ldots \\
\mathrm{A}_{\mathrm{n}} \\
\mathrm{B}_{0} \\
\ldots \\
\mathrm{B}_{\mathrm{n}-1} \\
\mathrm{D}
\end{array}\right], \\
\mathrm{XY}=\left[\begin{array}{ccccccc}
\mathrm{F}_{0 \mathrm{x}, 1} \cdot \mathrm{x}_{1} & \ldots & \mathrm{F}_{\mathrm{nx}, 1} \cdot \mathrm{x}_{1}^{(\mathrm{n})} & \mathrm{F}_{0 \mathrm{y}, 1} \cdot \mathrm{y}_{1} & \ldots & \mathrm{F}_{\mathrm{ny}, 1} \cdot \mathrm{y}_{1}^{(\mathrm{n}-1)} & 1 \\
\mathrm{~F}_{0 \mathrm{x}, 2} \cdot \mathrm{x}_{2} & \ldots & \mathrm{F}_{\mathrm{nx}, 2} \cdot \mathrm{x}_{2}^{(\mathrm{n})} & \mathrm{F}_{0 \mathrm{y}, 2} \cdot \mathrm{y}_{2} & \ldots & \mathrm{F}_{\mathrm{ny}, 2} \cdot \mathrm{y}_{2}^{(\mathrm{n}-1)} & 1 \\
\ldots & \ldots & \ldots & \ldots & \ldots & \ldots & \ldots \\
\mathrm{F}_{0 \mathrm{x}, \mathrm{N}} \cdot \mathrm{x}_{\mathrm{N}} & \ldots & \mathrm{F}_{\mathrm{nx}, \mathrm{N}} \cdot \mathrm{x}_{\mathrm{N}}^{(\mathrm{n})} & \mathrm{F}_{0 \mathrm{y}, \mathrm{N}} \cdot \mathrm{y}_{\mathrm{N}} & \ldots & \mathrm{F}_{\mathrm{ny}, \mathrm{N}} \cdot \mathrm{y}_{\mathrm{N}}^{(\mathrm{n}-1)} & 1
\end{array}\right]
\end{array}
$$

The system (58) with respect to some or all parameters $A_{j}, B_{j}$ and $D$ can be non-linear or quite linear. In case of nonlinearity, non-linear optimisation has to be applied, for instance by the simplex search method. An initial estimation of the parameters can be carried out either by omitting nonlinearities, if this is possible, or by using another estimation procedure. It is advisable to try several sets of starting values to make sure that the solution gives relatively consistent results. The obtained result for a given optimal averaging width $\mathrm{d}_{\mathrm{opt}}$ of the measurement window $\mathrm{g}(\mathrm{v})$ does not have to be an optimum. The verification of the result of the identification, the model parameters, can be done through the 
determination of the mean square deviation between the output signal $y$ and the model response obtained through the simulation $\mathrm{y}_{\mathrm{s}}$ for the assigned parameters:

$$
\mathrm{J}=\sum_{\mathrm{i}=1}^{\mathrm{N}}\left(\mathrm{y}_{\mathrm{s}}-\mathrm{y}\right)^{2}=\left\|\mathrm{y}_{\mathrm{s}}-\mathrm{y}\right\|^{2} .
$$

Both signals $y$ and $y_{s}$ must be averaged and corrected. The minimum of criteria J proves the result of non-linear optimisation and correctness of the choice of $\mathrm{d}_{\mathrm{opt}}$.

\subsection{Nonparametric identification of nonlinear dynamics of $1^{\text {st }}$ order of systems based on the active experiment (Boćkowska \& Żuchowski, 2007)}

Despite the fact that the presented method is nonparametric, its usefulness is limited to systems dynamics with structure described by the following differential equation:

$$
y^{(1)} \cdot \varphi(x, y)+y \cdot f(x, y)=x .
$$

Thanks to the fact that the measured window is an even function, the method of the averaged differentiation does not introduce any time shift between signals: the signals measured for example $y^{(1)}\left(t_{0}\right)$ and those who were averaged $y^{(1)}\left(t_{0}\right)_{g}$. The corrected signals are not shifted in relation to the measured signals, because the correction procedure is also even.

An active experiment ensures that a rich spectrum signal is fed into the system input. A rich spectrum can be achieved when a periodic signal with a modulated amplitude and frequency is applied, for example:

$$
\mathrm{x}(\mathrm{t})=\mathrm{A}(\mathrm{t}) \cdot \sin \left(\mathrm{t} \cdot \omega(\mathrm{t})+\phi_{0}\right) .
$$

Averaged and corrected courses of signals $x(t), y(t)$ and $y^{(1)}(t)$ should be obtained and the plots of these courses created. The time moments for which $y^{(1)}(t)=0$ can be easily obtained and correspond to the points of the static characteristics of the examined object:

$$
y \cdot f(x, y)=x .
$$

Next, the values of the second unknown function $\varphi(t)$ can be defined using all the values of both averaged and corrected signals.

The goal of the presented method is not the determination of the static characteristic, but that of the function $f(x, y)$. These two can produce the same or similar graphs, but they are not always the same mathematically. It means that the structure of the function $f(x, y)$ can not be concluded from the relation $\mathrm{y}(\mathrm{x})$ without additional assumptions. The functions $\mathrm{f}$ and $\varphi$ can be the functions only of $x$ or $y$ or of both signals together. Hence, various models can be created and some of them differ from the true model. The regression method should be used in order to determine the functions $f$ and $\varphi$ on the basis of the plots of the following relations:

$$
f(x, y)=z_{1}=\frac{x}{y}, \quad \varphi(x, y)=z_{2}=\frac{x-y \cdot f(x, y)}{y^{(1)}}
$$

in the co-ordinate system $x, y, z_{1}$ and $x, y, z_{2}$. An input with a rich spectrum enables to obtain a big collection of different points $x, y, z_{1}, z_{2}$. 
The examined object can have the dynamics of an order higher than one. In this case the obtained static characteristic is different from the real one and the change of the form of the signal $x(t)$ leads to other results. Hence, it is a good control test of the correctness of the dynamics order.

The identified model should be obviously verified using numerical simulation. A comparison between simulation results and measured data should also be carried out.

\subsection{Nonparametric identification of nonlinear dynamics of $2^{\text {nd }}$ order of systems}

The consideration relates to the models of system dynamics with the following structure:

$$
\mathrm{y}^{(2)} \cdot \mathrm{F}_{2}\left(\mathrm{x}, \mathrm{y}, \mathrm{y}^{(1)}\right)+\mathrm{y}^{(1)} \cdot \mathrm{F}_{1}\left(\mathrm{x}, \mathrm{y}, \mathrm{y}^{(1)}\right)+\mathrm{F}_{0}\left(\mathrm{x}, \mathrm{y}, \mathrm{y}^{(1)}\right)=\mathrm{x} .
$$

The averaged corrected values of signals $\mathrm{x}, \mathrm{y}, \mathrm{y}^{(1)}$ and $\mathrm{y}^{(2)}$ are used. At first the relation corresponding to static characteristics should be determined - a steady state, when $y^{(1)}(t)=0$, $\mathrm{y}^{(2)}(\mathrm{t})=0$ and $\mathrm{x}(\mathrm{t})=\mathrm{x}=$ const.:

$$
\mathrm{F}_{0}(\mathrm{x}, \mathrm{y}, 0)=\mathrm{x}
$$

Usually the function $F_{0}(x, y)$ is independent at $y^{(1)}(t)$, hence the substitute relation can be obtained:

$$
\mathrm{y}=\phi(\mathrm{x})
$$

Next, the values of $x, y$ corresponding to the extreme values of the first derivative should be chosen, for which:

$$
\mathrm{y}^{(2)}(\mathrm{t})=0 .
$$

The knowledge of the function $\mathrm{F}_{0}(\mathrm{x}, \mathrm{y})$, which was determined in the first step and multiple chosen values of $\mathrm{y}^{(1)}$ ex in the second step allow us to evaluate the structure of the function $\mathrm{F}_{1}\left(\mathrm{x}, \mathrm{y}, \mathrm{y}^{(1)}\right)$ :

$$
F_{1}\left(x, y, y^{(1)}\right)=\frac{x-F_{0}(x, y)}{y^{(1)} e x} .
$$

In a particular case, if $F_{1}\left(x, y, y^{(1)}\right)$ is the function only of the output, the relation (68) is obtained uniquely:

$$
F_{1}(y)=\frac{x-F_{0}(x, y)}{y^{(1)} e x} .
$$

In different cases the additional conditions should be assumed a priori.

The finding of the values of $x, y$ and $y^{(2)}$ for $y^{(1)}(t)=0$ allows us to choose the structure of the last function:

$$
F_{2}(y)=\frac{x-F_{0}(x, y)}{y^{(2)}} .
$$

The result of non-parametric identification is unique if the model of system dynamics can be described by the following differential equation: 


$$
\mathrm{y}^{(2)} \cdot \mathrm{F}_{2}(\mathrm{y})+\mathrm{y}^{(1)} \cdot \mathrm{F}_{1}(\mathrm{y})+\mathrm{F}_{0}(\mathrm{y})=\mathrm{x} .
$$

The accuracy of this process is defined by the accuracy of the operation of averaged differentiation with correction.

\subsection{Nonparametric identification of nonlinear dynamics of order higher than $2^{\text {nd }}$}

For the systems, which model has the analogue structure to (71) but is the higher order the parametric identification is necessary. Let us now consider:

$$
\mathrm{y}^{(3)} \cdot \mathrm{F}_{3}(\mathrm{y})+\mathrm{y}^{(2)} \cdot \mathrm{F}_{2}(\mathrm{y})+\mathrm{y}^{(1)} \cdot \mathrm{F}_{1}(\mathrm{y})+\mathrm{F}_{0}(\mathrm{y})=\mathrm{x}
$$

After the determination of the static characteristics and definition of the relation:

$$
\mathrm{F}_{0}(\mathrm{y})=\mathrm{x}
$$

the values of $x, y$ corresponding to the extreme values of the first or second derivative should be chosen, because both of these conditions are fulfilled very rarely. Supposing that $y^{(3)}(t)=0$ we obtain:

$$
\mathrm{y}^{(2)} \mathrm{ex} \cdot \mathrm{F}_{2}(\mathrm{y})+\mathrm{y}^{(1)} \cdot \mathrm{F}_{1}(\mathrm{y})=\mathrm{x}-\mathrm{F}_{0}(\mathrm{y})
$$

The structure of the functions $F_{2}$ and $F_{1}$ can be assumed as follows:

$$
F_{2}(y)=\sum_{i=0}^{n} a_{i} \cdot y^{i}, \quad F_{1}(y)=\sum_{i=0}^{m} b_{i} \cdot y^{i}
$$

and obtained using the regression. However if $\mathrm{y}^{(2)}(\mathrm{t})=0$ we obtain:

$$
\mathrm{y}^{(3)} \mathrm{ex} \cdot \mathrm{F}_{3}(\mathrm{y})+\mathrm{y}^{(1)} \cdot \mathrm{F}_{1}(\mathrm{y})=\mathrm{x}-\mathrm{F}_{0}(\mathrm{y})
$$

and the function $F_{1}$ can be treated as determined. Consequently, the form of the function $F_{3}$ can be found. In case of models of a higher order the procedure can be analogous.

\subsection{An optimal degree of complexity of a model}

In all the cases described above the analytical form of the functions $F_{0}, F_{1}, \ldots F_{k}$ is evaluated using the appropriate set of data, supporting the base functions:

$$
\mathrm{F}_{\mathrm{k}}(\mathrm{y})=\sum_{\mathrm{i}=0}^{\mathrm{n}} \mathrm{c}_{\mathrm{i}} \cdot \mathrm{f}_{\mathrm{i}}(\mathrm{y})
$$

and regression method remembering that the variables $\mathrm{x}$ and $\mathrm{y}$ are known with the certain accuracy $\Delta x$ and $\Delta y$. If the measured accuracy of variables can be obtained the optimal degree of complexity of the model can be determined. Assuming that the model with the degree of complexity $n$ in the form:

$$
F_{n}(y)=\sum_{i=0}^{n} c_{i} \cdot y^{i}
$$


was determined by regression based on the minimisation of the error:

$$
D^{2}\left(c_{i}\right)=\int_{y}\left\{F(y)-\sum_{i=0}^{n} c_{i} \cdot y^{i}\right\}^{2} \cdot d y
$$

hence the following equations are satisfied for $\mathrm{i}=0,1, \ldots, \mathrm{n}$ :

$$
\frac{\partial D^{2}\left(c_{i}\right)}{\partial c_{i}}=0=-2 \cdot \int_{y}\left\{F(y)-\sum_{i=0}^{n} c_{i} \cdot y^{i}\right\} \cdot y^{i} \cdot d y .
$$

If the values of $y$ are known with the accuracy $\Delta y=$ const or $\Delta_{1}=\Delta y / y=$ const then the application of the model (78) is connected with the additional error, for the small $\Delta y$ defined by the relation:

$$
F_{n}(y+\Delta y)=F_{n}(y)+\Delta y \cdot \frac{d F_{n}(y)}{d y}=F_{n}(y)+\Delta y \cdot \sum_{i=0}^{n} i \cdot c_{i} \cdot y^{i-1}
$$

or

$$
F_{n}(y+\Delta y)=F_{n}(y)+\Delta y \cdot \frac{d F_{n}(y)}{d y}=F_{n}(y)+\Delta 1 \cdot \sum_{i=0}^{n} i \cdot c_{i} \cdot y^{i}
$$

The square error is as follows:

$$
D_{a}^{2}(n)=(\Delta \mathrm{y})^{2} \int_{y}\left\{\sum_{i=0}^{n} c_{i} \cdot y^{i-1}\right\}^{2} \cdot d y \quad \text { or } \quad \mathrm{D}_{\mathrm{a}}{ }^{2}(\mathrm{n})=\Delta_{1}{ }^{2} \int\left\{\sum_{\mathrm{y}}^{\mathrm{n}} \mathrm{c}_{\mathrm{i}} \cdot \mathrm{y}^{\mathrm{i}}\right\}^{2} \cdot \mathrm{dy},
$$

A total model error:

$$
\mathrm{D}^{2}(\mathrm{n})=\mathrm{D}_{\min }{ }^{2}(\mathrm{n})+\mathrm{D}_{\mathrm{a}}{ }^{2}(\mathrm{n}),
$$

depends on its degree of complexity $\mathrm{n}$. If for the supported $\Delta \mathrm{y}$ or $\Delta_{1}$ as a result of the calculations one gets $D^{2}(n+1) \geq D^{2}(n)$, then the degree of model complexity $n$ is optimal.

\section{Conclusion}

The presented method can be useful in identification of the structure of a model of nonlinear dynamics of 1st and higher orders. The advantage of the proposed solution is its simplicity and possibility of evaluation of its accuracy. The application of the averaged differentiation with correction ensures one to evaluate an averaged signal and its derivatives close to their real values. A novelty is the proposed procedure of the correction of the averaged differentiation. The method allows to obtain a structure of the model of the nonlinear dynamics of $1^{\text {st }}$ or $2^{\text {nd }}$ order. The advantage of this identification is the fact that it can be used even if the measured signals are disturbed and the dynamics is nonlinear. In case of 
dynamics of a higher order the application of parametric identification to define a part of model structure is the solution.

\section{References}

Billings, S.A. \& Tsang, K.M. (1992). Reconstruction of linear and non-linear continuous time models from discrete time sampled-data systems, Mech. Systems and signal Processing, Vol. 6, No. 1, pp. 69-84

Boćkowska, M. (1998). Reconstruction of input of a measurement system with simultaneous identification of its dynamics in the presence of intensive, random disturbances, Ph.D. thesis, Technical University of Szczecin

Boćkowska, M. (2003). Application of the averaged differentiation method to the parameter estimation of non-linear systems. Proceedings of the 9th IEEE International Conference on Methods and Models in Automation and Robotics, pp. 695-700, Technical University of Szczecin, Międzyzdroje, 2003

Boćkowska, M. (2005). Corrector design for the window-fft processing during the parametric identification of non-linear systems, Proceedings of the 11th IEEE International Conference on Methods and Models in Automation and Robotics, pp. 461466, Technical University of Szczecin, Międzyzdroje, 2005

Boćkowska, M. (2006). Identification of system dynamics using the averaged differentiation with correction adapted to output spectrum, Proceedings of the 12th IEEE International Conference on Methods and Models in Automation and Robotics, pp. 461466, Technical University of Szczecin, Międzyzdroje, 2006

Boćkowska, M. \& Żuchowski, A. (2007). Application of the averaged differentiation method to the parameter estimation of non-linear systems, Proceedings of the 13th IEEE International Conference on Methods and Models in Automation and Robotics, pp. 461466, Technical University of Szczecin, Szczecin, 2007

Eykhoff, P. (1980). Identification in dynamics systems, PWN, Warsaw

Greblicki, W. \& Pawlak, M. (1994). Cascade nonlinear system identification by a nonparametric method, Int. Journal of System Science, Vol. 25, No 1, pp. 129-153

Haber, R. \& Keviczky, L. (1999). Nonlinear system identification - input - output modelling approach. Kluwer Academic Publishers

Iserman, R. (1982). Identifikation dynamischer Systeme, Springer Verlag, Berlin

Kordylewski, W. \& Wach, J. (1988). Averaged differentiation of disturbed measurement signals. PAK, No. 6

Nuttall, A.H. (1981). Some windows with very good sidelobe behaviour. IEEE Trans. On Acoustic, Speech and Signal Processing 29, No 1, pp. 84-91

Söderström, V.T. \& Stoica, P. (1989). System identification, Englewood Clifs, NJ: Prentice Hall

Uhl, T. (1997). Computer aided identification of models of mechanical constructions, WNT, Warsaw 


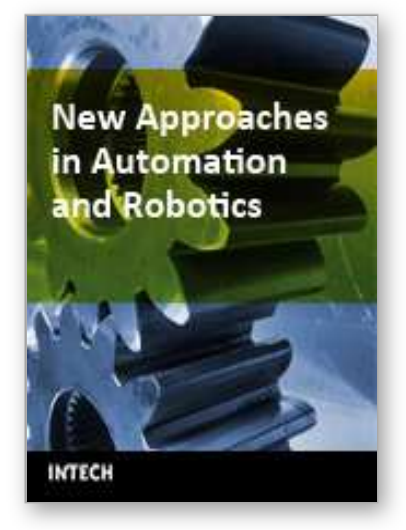

\author{
New Approaches in Automation and Robotics \\ Edited by Harald Aschemann
}

ISBN 978-3-902613-26-4

Hard cover, 392 pages

Publisher I-Tech Education and Publishing

Published online 01, May, 2008

Published in print edition May, 2008

The book New Approaches in Automation and Robotics offers in 22 chapters a collection of recent developments in automation, robotics as well as control theory. It is dedicated to researchers in science and industry, students, and practicing engineers, who wish to update and enhance their knowledge on modern methods and innovative applications. The authors and editor of this book wish to motivate people, especially under-graduate students, to get involved with the interesting field of robotics and mechatronics. We hope that the ideas and concepts presented in this book are useful for your own work and could contribute to problem solving in similar applications as well. It is clear, however, that the wide area of automation and robotics can only be highlighted at several spots but not completely covered by a single book.

\title{
How to reference
}

In order to correctly reference this scholarly work, feel free to copy and paste the following:

Magdalena Bockowska and Adam Zuchowski (2008). Nonparametric Identification of Nonlinear Dynamics of Systems Based on the Active Experiment, New Approaches in Automation and Robotics, Harald Aschemann (Ed.), ISBN: 978-3-902613-26-4, InTech, Available from:

http://www.intechopen.com/books/new_approaches_in_automation_and_robotics/nonparametric_identification _of_nonlinear_dynamics_of_systems_based_on_the_active_experiment

\section{INTECH}

open science | open minds

\author{
InTech Europe \\ University Campus STeP Ri \\ Slavka Krautzeka 83/A \\ 51000 Rijeka, Croatia \\ Phone: +385 (51) 770447 \\ Fax: +385 (51) 686166 \\ www.intechopen.com
}

\author{
InTech China \\ Unit 405, Office Block, Hotel Equatorial Shanghai \\ No.65, Yan An Road (West), Shanghai, 200040, China \\ 中国上海市延安西路65号上海国际贵都大饭店办公楼 405 单元 \\ Phone: +86-21-62489820 \\ Fax: +86-21-62489821
}


(C) 2008 The Author(s). Licensee IntechOpen. This chapter is distributed under the terms of the Creative Commons Attribution-NonCommercialShareAlike-3.0 License, which permits use, distribution and reproduction for non-commercial purposes, provided the original is properly cited and derivative works building on this content are distributed under the same license. 